\title{
Understanding the functions and relationships of the glymphatic system and meningeal lymphatics
}

\author{
Antoine Louveau, ${ }^{1}$ Benjamin A. Plog, ${ }^{2}$ Salli Antila, ${ }^{3}$ Kari Alitalo, ${ }^{3}$ Maiken Nedergaard, ${ }^{2,4}$ and Jonathan Kipnis \\ ${ }^{1}$ Center for Brain Immunology and Glia, Department of Neuroscience, School of Medicine, University of Virginia, Charlottesville, Virginia, USA. ${ }^{2}$ Center for Translational Neuromedicine, University of Rochester \\ Medical Center, Rochester, New York, USA. ${ }^{3}$ Wihuri Research Institute and Translational Cancer Biology Program, Biomedicum Helsinki, University of Helsinki, Helsinki, Finland. ${ }^{4}$ Center of Neuroscience, \\ Faculty of Health and Medical Sciences, University of Copenhagen, Copenhagen, Denmark.
}

\begin{abstract}
Recent discoveries of the glymphatic system and of meningeal lymphatic vessels have generated a lot of excitement, along with some degree of skepticism. Here, we summarize the state of the field and point out the gaps of knowledge that should be filled through further research. We discuss the glymphatic system as a system that allows CNS perfusion by the cerebrospinal fluid (CSF) and interstitial fluid (ISF). We also describe the recently characterized meningeal lymphatic vessels and their role in drainage of the brain ISF, CSF, CNS-derived molecules, and immune cells from the CNS and meninges to the peripheral (CNS-draining) lymph nodes. We speculate on the relationship between the two systems and their malfunction that may underlie some neurological diseases. Although much remains to be investigated, these new discoveries have changed our understanding of mechanisms underlying CNS immune privilege and CNS drainage. Future studies should explore the communications between the glymphatic system and meningeal lymphatics in CNS disorders and develop new therapeutic modalities targeting these systems.
\end{abstract}

Perivascular pathways: pseudolymphatic vessels of the brain?

In peripheral organs, colloids are extravasated across a fenestrated capillary bed, and these proteins, as well as excess tissue fluid, are returned to the circulating blood through a dense network of lymphatic vessels $(1,2)$. In the CNS, the blood-brain barrier prevents unregulated protein and fluid entry into brain $(3,4)$; however, soluble waste products are still released into the brain's interstitial spaces as a consequence of its high metabolic rate. The CNS is the only organ system of the body lacking classically defined lymphatic vessels and tissues, and as a result has developed unique adaptations for waste clearance and fluid balance (5). Choroid plexus epithelial cells actively secrete cerebrospinal fluid (CSF) within the cerebral ventricles $(4,6-8)$, ultimately draining into the subarachnoid space (SAS) surrounding the brain, which has long been considered to act as a sink for interstitial waste $(6,9)$. How metabolites produced and released deep within the brain's extracellular spaces ultimately clear to the subarachnoid CSF compartment remains a persistent question (7-9). Early studies of the anatomy of brain waste clearance used tracer molecules to identify pathways of interstitial fluid (ISF) and solute drainage from the parenchyma. Injection of Blue Dextran 2000 or horseradish peroxidase (HRP) directly into the caudate nucleus of rats showed that these interstitial solutes did not move through the parenchyma isotropically, but rather drained via preferential pathways, specifically the perivascular spaces of cere-

Authorship note: A. Louveau, B.A. Plog, and S. Antila contributed equally to this work. K. Alitalo, M. Nedergaard, and J. Kipnis contributed equally to this work and are co-senior authors.

Conflict of interest: The authors have declared that no conflict of interest exists. Reference information: / Clin Invest. 2017;127(9):3210-3219.

https://doi.org/10.1172/JCI90603. bral blood vessels $(10,11)$. Molecules of vastly different molecular weights, such as albumin $(69 \mathrm{kDa})$ and polyethylene glycols $(900$ $\mathrm{Da}$ and $4 \mathrm{kDa}$ ), all cleared from brain with nearly identical halftimes of disappearance, leading to the conclusion that the efflux of ISF and its constituent solutes was by bulk fluid flow and not diffusion (12). Similarly, HRP injected into the CSF of cats was found to use these same perivascular conduits to rapidly enter the brain, penetrating to the level of capillary basement membranes within 4 to 10 minutes of injection $(13,14)$. Consequently, a model was advanced in which the brain's perivascular spaces were assigned a pseudolymphatic function, acting as ducts to remove metabolic waste products and deliver fresh CSF to the interstitium.

\section{The glymphatic system: anatomy and function} in health and disease

Observations that ISF and CSF were moving in opposite directions within this exchange pathway led to the hypothesis that there are discrete subsets of perivascular spaces specialized for either ISF efflux or CSF influx. In an attempt to identify the inflow and clearance arms of this system, Iliff and colleagues (15) injected fluorescent tracers into the CSF and striatum of Tie2-GFP:NG2-DsRed double reporter mice to visualize all cerebral blood vessels based on GFP-expressing endothelial cells (ECs) and DsRed-expressing perivascular cells (15). Here they observed that CSF-based tracer entered the brain specifically within the perivascular spaces of double-positive vessels, indicative of cerebral-penetrating arteries, whereas ISF tracer drained from brain exclusively alongside single-positive large caliber veins, such as the internal cerebral and caudal rhinal veins (15). Additionally, perivascular aquaporin-4 (AQP4) channels, densely expressed in astrocyte end-foot processes (16), were found to play an important role in facilitating flow through this pathway. 
A

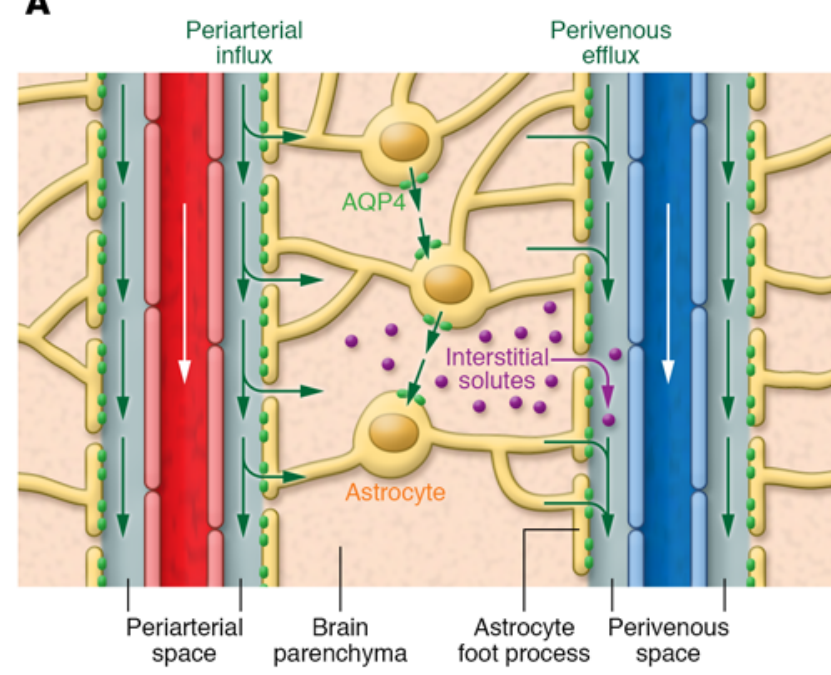

B

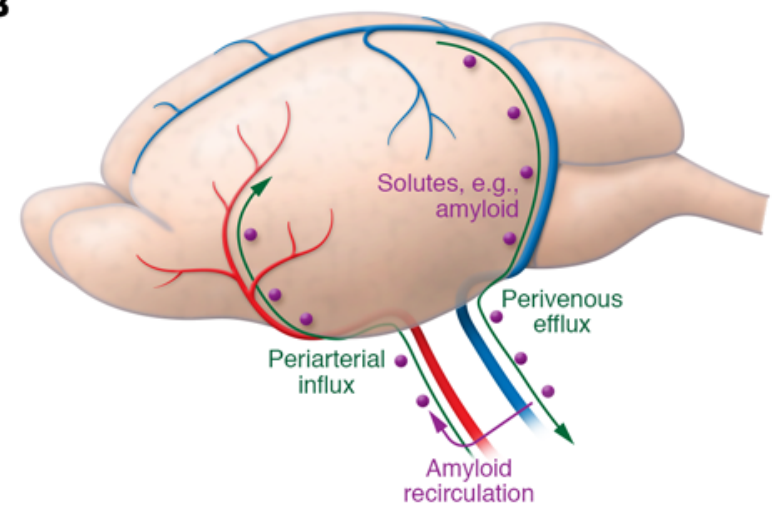

Figure 1. Circulation of CSF, ISF, and brain solute through the glymphatic pathway. (A) CSF within the subarachnoid and cisternal spaces flows into brain specifically via periarterial spaces and then exchanges with ISF facilitated by aquaporin-4 (AQP4) water channels that are positioned within perivascular astrocyte end-foot processes. The bulk movement of CSF into brain drives the convective flow of ISF and interstitial solute through the extracellular space to ultimately collect within perivenous spaces. (B) Perivenous fluid and solute then drain from brain predominantly alongside large-caliber ventral veins. Once within the subarachnoid CSF, solutes such as amyloid- $\beta$ can exit the cranium via arachnoid granulations or meningeal lymphatic vessels or along cranial and spinal nerves; however, a proportion is also capable of recirculating into brain via periarterial spaces. Periarterial solute may seed and accumulate within the basement membranes of smooth muscle cells, precipitating conditions such as cerebral amyloid angiopathy.

knockout, CSF volume reduction, or alteration of posture all led to increased brain and reduced cervical lymph node lactate levels (21), even in the clearance-promoting anesthetized state (20). The importance of glymphatic clearance of interstitial solute is perhaps best demonstrated by the fact that its failure contributes to the pathophysiology of various CNS diseases. In the aged brain and in a murine transgenic model of Alzheimer's disease, glymphatic CSF influx is reduced and the clearance of $A B$ is profoundly impaired $(22,23)$. Interestingly, pretreating young wild-type mice with $A \beta$ suppressed glymphatic influx, suggesting that not only is $A \beta$ aggregation a consequence of a breakdown of flow within this pathway, but it may also be an independent cause of glymphatic dysfunction (23). Further, glymphatic impairment is a prominent feature of cerebrovascular disease, including both subarachnoid hemorrhage and multiple microinfarction $(24,25)$. Following traumatic brain injury, reduced clearance of cortical interstitial solute was associated with motor and cognitive neurological deficits (26). While CSF-ISF exchange within the glymphatic pathway is improved by increased cerebral penetrating arterial pulsatility (27), the sleep state (20), and a lateral head position during sleep (28), none of these regulatory factors have yet been demonstrated to improve waste clearance in any of the above disease states. Consequently, a critical area of future glymphatic research is identification of novel mechanisms to control flow through this pathway with the goal of modifying diseases characterized by toxic extracellular solute accumulation.

\section{Is $A \beta$ cleared along the periarterial space?}

Cerebral amyloid angiopathy is a disease process characterized by accumulation of soluble $\mathrm{A} \beta$ within the walls of cerebral arteries, ultimately weakening the integrity of these vessels and leading to lobar hemorrhage (29). Based on this observation, Weller, Carare, and colleagues hypothesized that, contrary to the glymphatic model, interstitial waste clears from brain within an arterial intramural pathway (30). Using ex vivo fluorescence and electron microscopy, this group demonstrated that tracer molecules injected directly into brain appear within the basement membranes of cerebral capillaries and smooth muscle cells of the arterial tunica media (30). These tracer imaging experiments were qualitative, making it difficult to determine whether periarterial efflux was a major phenomenon. Additionally, it is unknown whether this flow pattern is specific to the striatum, where all tracer injections were performed, or rather more broadly characteristic of the entirety 
of the cerebrum. Further, it is possible that there was retrograde flow of tracer along the cannula track into the subarachnoid CSF, suggesting that periarterial tracer was due to glymphatic CSF influx rather than ISF efflux. Finally, as shown in a recent study by Bedussi and colleagues, the appearance of tracer in a periarterial distribution may result from pressure injection of tracer into brain tissue immediately adjacent to an artery, rather than occurring as a consequence of true ISF bulk flow (31). Based on these findings, however, it was postulated that once within the arterial wall, parenchymal solutes travel in a direction opposite to blood flow toward larger and more proximal vessels until they ultimately reach the internal cerebral artery (ICA), where solute can be drained by deep cervical lymph nodes (dcLNs) $(30,32,33)$. While $\mathrm{A} \beta$ has been detected within the walls of intracerebral arteries, including those of young individuals (34), intramural $\mathrm{A} \beta$ has never been identified within the ICA immediately outside the skull (32), challenging the notion that solutes use periarterial pathways to exit the cranium, and instead suggesting that arterial walls may be vulnerable to metabolite accumulation.

As the above-discussed experiments were all conducted ex vivo lacking temporal resolution, it is difficult to conclude that periarterial spaces are the most immediate site of parenchymal solute drainage. Rather, it is equally possible that waste accumulation around arteries represents a recirculation event, where interstitial metabolites, including $A \beta$, first clear via glymphatic perivenous spaces to the subarachnoid CSF compartment. Subsequently, $A \beta$ reenters brain within periarterial spaces, and begins depositing after coming in contact with the adhesive smooth muscle and capillary basement membranes. Like that seen in other small vessel diseases, such as CADASIL (35), altered protein expression in periarterial spaces may increase molecular affinity for $A \beta$ and thereby precipitate pathological accumulation.

Finally, to further bolster the concept of intramural, periarterial clearance, one study suggests that, as a result of close compaction of astrocyte end-foot processes, the glial basement membrane, pial membrane, and outer smooth muscle basement membrane, perivascular spaces do not exist within either the murine or the human cortex (33). However, data from this same group seem to refute this contention, demonstrating the presence of fluorescent microspheres ranging from $20 \mathrm{~nm}$ to $100 \mu \mathrm{m}$ in size within perivascular distributions (30). Additionally, Iliff and colleagues identified CSF-based tracer within both the endothelial basement membrane and the perivascular space between the arterial smooth muscle and the astrocyte end-foot of the glial limiting membrane (15). More recent studies propose a model of bidirectional fluid and solute movement within periarterial spaces CSF influx within the outer glymphatic pathway and ISF efflux via the inner intramural pathway (32). Computational modeling studies support this idea, suggesting that the orientation of basement membrane structures and reflection of the cardiac pulse wave at arterial branch points may play a role in promoting this reverse transport process $(36,37)$, but at present there are no empirical data to support either mechanism. It is here important to note that no tracers have been observed in the periarterial space moving against the direction of blood flow or the pulsatility of the vascular wall in 2-photon live-imaging experiments. Similarly, the computational modeling studies have suggested that glymphatic trans- port of solutes is mediated by diffusion (36-38). These reports are, however, contradicted by the classical studies from Cserr's group, which observed that transport velocity of intraparenchymal tracers was independent of their molecular size and therefore concluded that solute transport is mediated by convective flow (10-12). A more inclusive and validated model must therefore be established under healthy and pathological conditions to further demonstrate the contribution of periarterial and perivenous drainage.

\section{Anatomy of lymphatic vessels in the dura mater}

The lymphatic vascular network is a unique blind-ended unidirectional absorptive and transport system that penetrates most human tissues. It consists of draining lymphatic vessels, lymph nodes, and associated lymphatic organs. In mammalian embryos, the lymphatic organ system develops when the cardiovascular system has already started to function $(39,40)$. Unlike blood vessels, the lymphatic vessels do not form a closed circulatory network. In peripheral organs, lymphatic drainage contributes considerably to tissue fluid homeostasis and immune surveillance by facilitating the clearance of excess interstitial tissue fluid, macromolecules, and immune cells from the interstitium back into blood circulation. In the lymphatic network, fluid, cells, and macromolecules are first absorbed by blind-ended lymphatic capillaries (the initial lymphatic vessels), and the flow proceeds via precollector and collector lymphatic vessels and lymph nodes, ending in the thoracic duct and the right lymphatic trunk, which deliver lymph into subclavian veins in the neck region (2).

The meninges that envelop the brain and spinal cord participate in forming the various fluid compartments of the CNS. They are composed of three layers ("maters"), termed pia, arachnoid, and dura. The innermost pial layer lines and invests the brain parenchyma, whereas dura is adherent to the skull. Although a few isolated lymphatic vessels were previously reported around the cranial nerves and dural blood vessels (41-43), the CNS and its meningeal linings were long believed to be devoid of a lymphatic vascular system, which has posed long-standing questions about brain waste clearance, ISF/CSF outflow mechanisms, and immune surveillance (44). Recent work has described the extensive meningeal lymphatic vessel network that serves macromolecular clearance and immune cell trafficking functions in the brain $(45,46)$. Characterization of the entire network of vessels in the dura mater overlying the skull bones was possible using lymphatic EC-specific fluorescent reporters and immunofluorescent staining. Lymphatic vessels located alongside the arteries, major venous sinuses, and cranial nerves appear to provide a pathway of drainage out from the cranium along these anatomical structures via the various foramina at the base of the skull $(45,46)$. Furthermore, lymphatic vessels under the olfactory bulb crossed the cribriform plate, where olfactory nerves travel through bone into the nasal mucosa. Interestingly, meningeal vessels were thus found in similar periarterial and perineural locations where ISF and CSF flow was previously observed (47-50). Compared with the superior portions of the skull, the dural lymphatic network in the basal parts was more extensive and contained valves whose function was not obvious, as fluid outflow from the head is ensured by gravity even without valves, which are needed in other parts of the body to prevent backflow in collecting lymphatic vessels. 
A
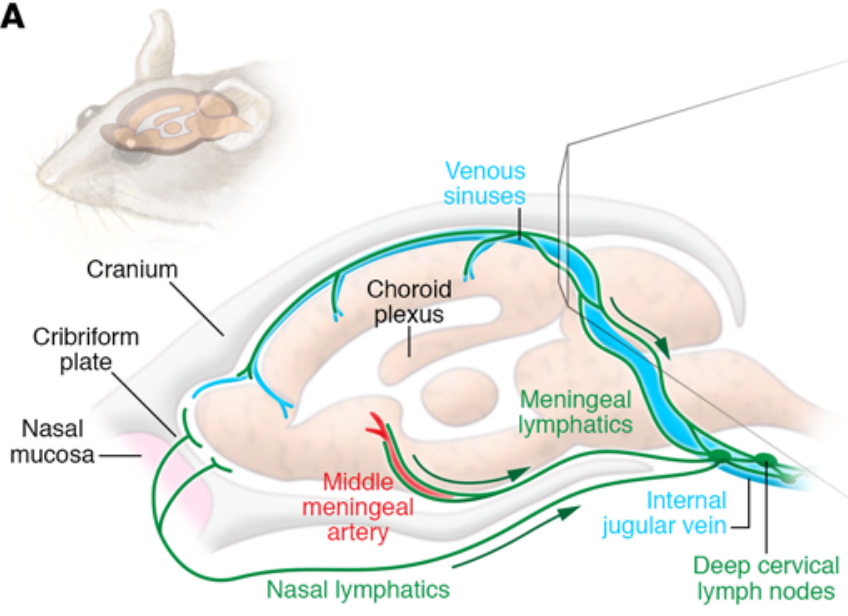

B

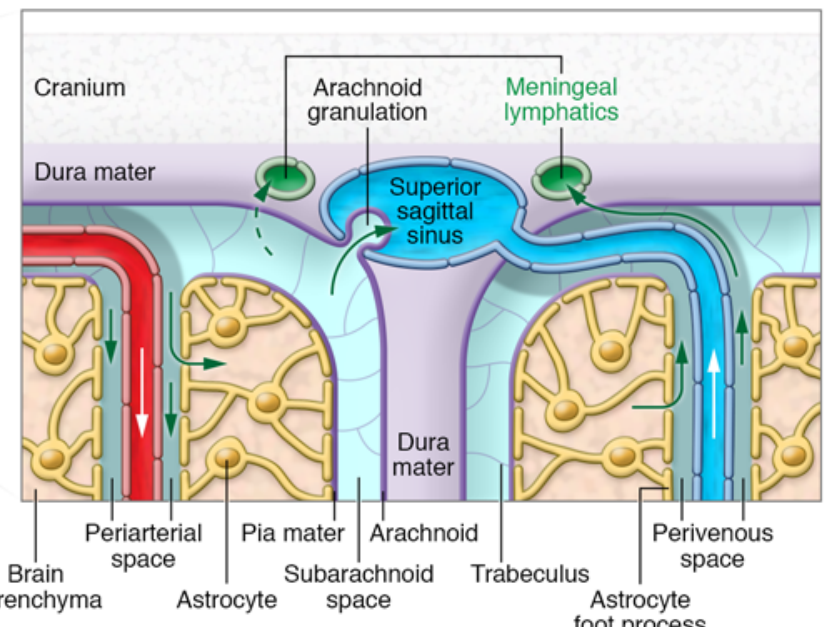

C
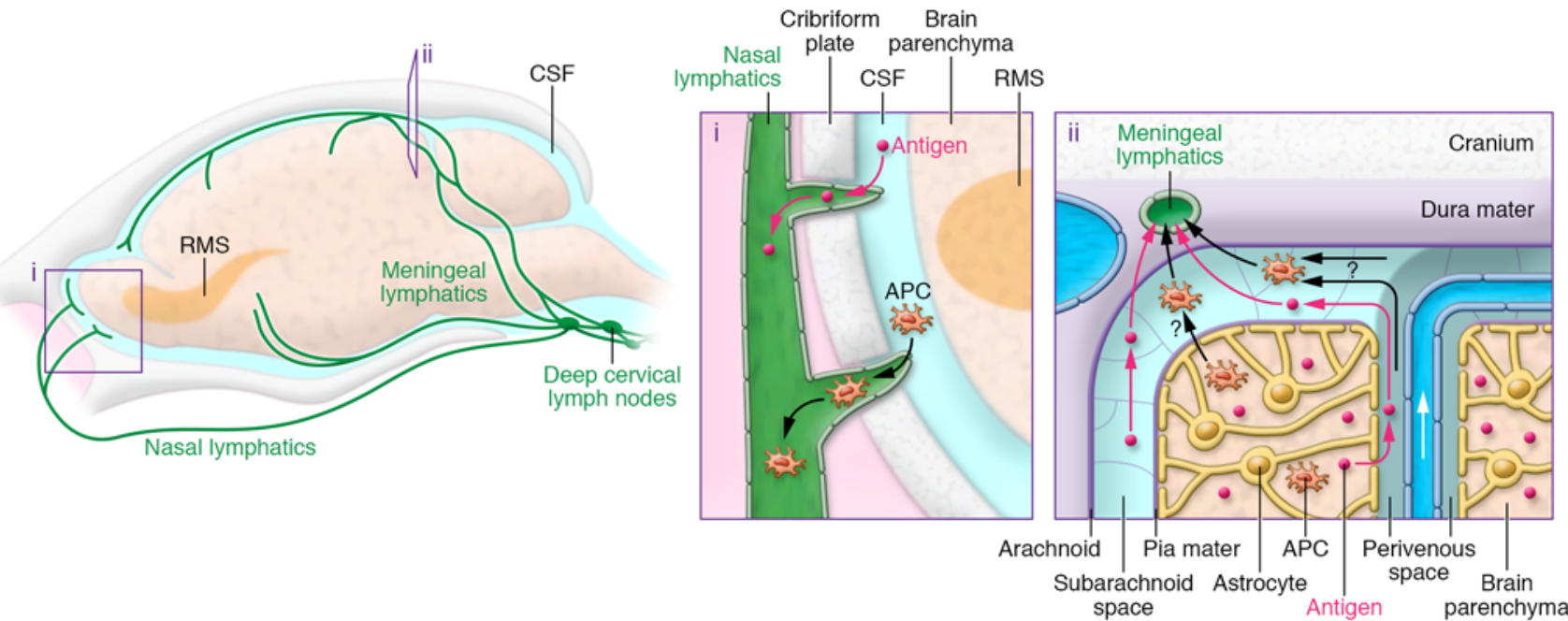

Figure 2. Glymphatic and lymphatic drainage pathways from the CNS to the cervical lymph nodes. (A) Schematic illustration of the meningeal lymphatic vessel system in mouse cranium. The dural lymphatic vessels align with dural blood vessels and cranial nerves and exit the cranium via the foramina together with the venous sinuses, arteries, and cranial nerves. Some lymphatic vessels are also found traversing the cribriform plate with the olfactory nerves. Tracers injected into either brain parenchyma or SAS drain via the dural lymphatic vessels into dcLNs located next to the jugular vein. (B) Close-up view of ISF and CSF circulation. The perivascular glymphatic drainage system transports CSF and solutes into the brain via a periarterial pathway, whereas ISF and solutes exit the brain via the perivenous glymphatic pathway. CSF can enter the venous system via arachnoid granulations, and CSF macromolecules and immune cells are transported mainly along the dural lymphatic vessels into the lymph nodes and extracranial systemic circulation. (C) Out-of-CNS drainage routes for antigens and antigen-presenting cells (APCs). Antigens and APCs are proposed to leave the CNS via either (i) lymphatics of the cribriform plate, reaching the nasal mucosa lymphatic vasculature (particularly, dendritic cells may migrate along the rostral migratory stream [RMS] to enter the lymphatics via the olfactory bulb's SAS); or (ii) the glymphatic pathway (as demonstrated for antigens), reaching the SAS and entering the meningeal lymphatic vasculature via SAS and trafficking to the dcLNs. APCs within the meningeal spaces may also leave through meningeal lymphatic vessels into the dcLNs. Each pathway's contribution to cell and antigen drainage has yet to be determined.

\section{Dural lymphatic vessels in ISF and CSF clearance}

According to previous studies, several different pathways contribute to circulation and extracranial clearance of CNS extracellular fluid compartments containing ISF and CSF. The perivascular exchange of fluid and solutes between ISF and CSF has been discussed in the previous sections. CSF produced by choroid plexuses in the brain ventricles and circulated into cranial and spinal SAS is considered to be drained via a dual outflow system consisting of the arachnoid granulations and the extracranial lymphatic system
(50). After the identification of arachnoid granulations over three centuries ago, various independent studies established their role in CSF outflow into the dural venous sinuses (51-54). Numerous groups reported the lymphatic clearance route after observing the transfer of macromolecules and tracers from brain parenchyma or CSF space into the extracranial lymphatic vasculature in various animal species (47, 50, 55-59). Until recently, clearance from the intracranial side was thought to occur via perineural sheaths of cranial and spinal nerves $(58,60,61)$. Observations of CSF trac- 
ers entering the lymphatic vessels of nasal mucous membranes through the cribriform plate suggested that the most important CSF outflow mechanism into extracranial lymphatic system would be via perineural SAS enveloping the olfactory nerve sheaths (47, 61-63). Besides cranial perineural flow, tracer studies also suggested that CSF from the spinal SAS would terminate in the regional lymphatics outside of the spinal canal via perineural pathways $(64,65)$. The latest addition into the ISF/CSF outflow system is the recently discovered meningeal lymphatic vasculature along the cranial nerves, arteries, and veins that traverse together through the cranial foramina and serve as a direct, continuous connection between the intracranial space and extracranial lymphatic vasculature (refs. 45, 46, and Figure 2, A and B).

Fluorescent tracer and macromolecule injections into different CNS compartments have elucidated the functionality of dural lymphatic vessels. Tracers injected into brain parenchyma and ventricles were observed along the meningeal lymphatic vessels and drained primarily into the dcLNs, but not into nonbrain-draining lymph nodes $(45,46)$. In a mouse model devoid of meningeal lymphatic vasculature, cerebral macromolecule clearance was attenuated and drainage into dcLNs was almost completely abrogated, suggesting direct clearance of macromolecules via meningeal lymphatic vessels into extracranial lymphatic tissue (46). Furthermore, surgical ligation of the lymphatic vessels emanating from dcLNs prevented tracer accumulation into the lymph node, led to increased meningeal T cell numbers, and enhanced tracer filling of the dural lymphatic network in the basal parts of the skull $(45,46)$. These observations further indicated a role for the dural lymphatic vessels in transporting intracranial cells, macromolecules, and ISF/CSF to the extracranial lymphatic system.

The location of the lymphatic vessels close to the SAS suggests that they absorb CSF from the arachnoid mater; however, the direct mechanism of fluid and macromolecular uptake into dural lymphatics is still unknown. It has been speculated that some of the CSF transported across the arachnoid barrier layer collects in the dural border interstitium and into "unlined channels" for further transportation or absorption into the endothelial-lined parasagittal plexus (50). Intraparenchymally injected tracers translocated to the dcLNs were also observed in the glymphatic space, suggesting a connection between the glymphatic and meningeal lymphatic systems (46). Considering that the glymphatic exit pathway from the brain interstitium is along veins (see "The glymphatic system: anatomy and function in health and disease" above) and that the dural lymphatics in many locations flank communicating veins, some glymphatics may be closely associated with absorptive lymphatic vessels, whose intercellular junctions are compatible with fluid entry.

Blockade of different outflow routes has demonstrated the importance of the lymphatic outflow route in maintaining normal intracranial fluid homeostasis. Surgical blockade of the perineural cribriform pathway was previously reported to cause rapid and sustained increase in intracranial pressure (66). Moreover, in dogs, acute interruption of lymphatic outflow by ligation of the cervical lymphatic vessels leading to cervical lymph nodes caused profound intracranial consequences, including a rise in intracranial pressure (47).
Cserr et al. estimated that between $14 \%$ and $47 \%$ of albumin tracer injected into the brain or CSF passes through the extracranial lymphatic system (59). However, the exact proportion of clearance along the meningeal lymphatics adjacent to blood vessels and spinal and cranial nerves is not yet known and may vary between species as well as in response to the functional dynamics of other outflow routes $(45,46)$. Arachnoid villi mature during late fetal and early postnatal periods and start to degenerate along with the choroid plexus in senescence (67-70), suggesting that adaptive changes in CSF clearance also occur via the different routes. Moreover, mice born without meningeal lymphatic vessels did not show any quantitative changes in brain interstitial fluid pressure or water content, further suggesting effective compensatory or adaptive mechanisms in the unchallenged state (46).

\section{CNS compartments and their immune connection}

The term "immune privilege" was coined by Peter Medawar to describe a tissue or organ in which the introduction of foreign antigen does not elicit an immune response. Experiments conducted by Medawar and colleagues in the late 1940s introduced the notion that the brain parenchyma is an immune-privileged site. Unlike similar grafts in the periphery, skin allografts implanted into the brain parenchyma elicit a delayed graft rejection (71). Less well known - though not less interesting - is that if an immune response toward allograft antigens has already been established in the periphery prior to grafting into brain parenchyma, the brain's immune response to the allograft will be similar to the peripheral organs' response (71). That finding suggests that although antigens within the brain do not prime an immune response, they can maintain and potentiate it. CNS immune privilege was attributed, in part, to a lack of lymphatic drainage from the CNS, with consequent failure to allow antigen presentation on professional antigen-presenting cells (such as dendritic cells). Interestingly, a regular immune response could be elicited when the allograft was implanted close to the ventricles or within the SAS, suggesting that compared with other brain areas, the meningeal spaces are more in touch with peripheral immunity $(72,73)$.

The brain's parenchymal and CSF/meningeal compartments possess different properties that confer on each a unique immune status. Under physiological conditions, numerous immune cells carry out CNS surveillance and occupy the CSF/meningeal compartment $(45,74-76)$. Meningeal ECs lack astrocytic end-feet and also differ in other ways from brain ECs. As a result, the blood-meningeal barrier is more permissive than the blood-brain barrier to entry of immune cells and allows them to circulate within the meninges under homeostatic conditions (77). The ability of such meningeal immune cells to penetrate the brain parenchyma under pathological conditions (assuming that this does occur physiologically) is still not fully understood (78).

Tracing studies have revealed marked differences between the draining properties of the meningeal and parenchymal compartments (Figure 2C). Macromolecules and tracers injected into the SAS rapidly reach the cervical lymph nodes, suggesting that CSF constituents can easily drain outside the CNS and thus potentially elicit an immune response. The different routes potentially implicated in such drainage were described earlier (see "Dural 


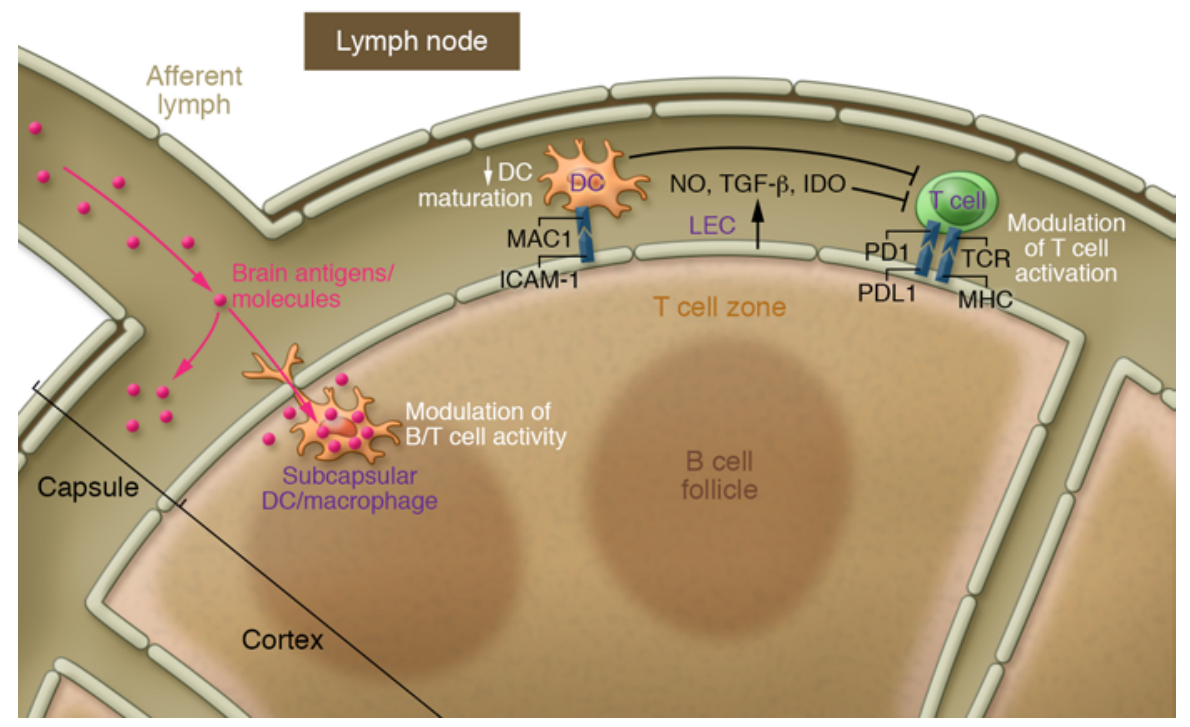

Figure 3. Potential modulation of $\mathrm{T}$ cell activation by lymphatic ECs. Lymphatic ECs (LECs) (primarily within the lymph nodes) can modulate T cell activation directly, either by producing modulatory molecules (NO, IDO, TCF- $\beta$ ) or by expressing $\mathrm{MHC}$ and costimulatory molecules like PDL1. Alternatively, the regulation can also be indirect by inducing maturation of dendritic cells (DCs) via ICAM-1 expression. Moreover, soluble molecules draining from the CSF to the dcLNs are taken up by subcapsular APCs (DCs and macrophages), resulting in modulation of $B$ and $T$ cell activation in the dcLNs. This function of meningeal lymphatic vessels in regulation of meningeal T cell tolerance has not yet been explored. IDO, Indoleamine 2,3-dioxygenase; PD1, programmed death 1; PDL1, Programmed death ligand 1.

lymphatic vessels in ISF and CSF clearance" above). Similarly to tracers, injection of immune cells into the SAS results in their accumulation in the CNS-draining but not in non-brain-draining lymph nodes (79).

Tracers injected into the brain parenchyma itself also drain into the cervical lymph nodes $(26,59)$. As discussed above, this connection is primarily due to the glymphatic system that connects ISF and CSF (15). In both rodent and primate models of multiple sclerosis, for example, brain antigens were increased in the cervical lymph nodes $(80,81)$. After stroke, brain injury, or oligodendrocyte death induced by diphtheria toxin, myelin antigens have similarly been found to accumulate in the cervical lymph nodes (82-84). Drainage of brain antigens has been observed in animal models not only of autoimmune diseases but also of Alzheimer's disease. $A \beta$, for example, was detected in the cervical lymph nodes in murine models of Alzheimer's disease (85).

Injection of immune cells into the brain parenchyma has yielded controversial findings. While some studies have followed injections of $\mathrm{T}$ cells, monocytes, or dendritic cells from the brain parenchyma to their ultimate drainage into the cervical lymph nodes, others have failed to document any such drainage after intracerebral injection (79, 86-89). Furthermore, the perivascular pathway is likely incapable of allowing the passage of immune cells (48), raising the possibility that at least some of the observed drainage might be due to postinjection leakage into the CSF/meningeal compartment. In yet other studies, the cribriform plate was proposed as the route by which immune cells exit the CNS and reach the nasal lymphatics $(87,88)$. Clearly, the notion that $\mathrm{T}$ cells or dendritic cells within the brain parenchyma have migratory potential to leave the parenchyma remains a matter of debate. Not only are microglia nonmigratory cells, but studies have also suggested that the brain parenchymal environment promotes the death of $\mathrm{T}$ cells and dendritic cells rather than recirculation (90, 91). Thus, recirculation of infiltrating immune cells from the brain parenchyma might not occur under physiological (or even pathological) conditions. Recent work suggests that endogenous $\mathrm{CD} 11 \mathrm{c}^{+}$ cells might recirculate from the CNS under neuroinflammatory conditions, but the exact provenance of such cells (meningeal or brain compartment) remains uncertain (92).
All in all, accumulating evidence indicates that the brain's parenchymal and CSF/meningeal compartments each possess distinctive drainage characteristics that endow them with unique immune properties.

\section{Communication between the CNS compartments}

While the brain and the meninges have distinctive drainage properties, they are not isolated from one another and can indeed communicate. Intermixing between the ISF and the CSF (as described in previous sections) reflects their direct contact. CSF and its constituents can act directly on brain function. Notably, vitamin C and folate diffuse into the brain through the $\operatorname{CSF}(93,94)$. Many other ions and proteins are transported through the choroid plexus epithelium and diffuse from the CSF into the brain, where they affect neuronal function (94). More recently, the immune compartment in the meninges was implicated in neuronal function (75). Notably, IL-4 and IL-13 production by meningeal T cells appears to contribute to the maintenance of cognitive function in mice $(74,95)$. Furthermore, production of IFN- $\gamma$ in the SAS directly regulates social behavior in mice (96).

Taken together, the evidence indicates that the brain and meningeal compartments are not isolated from one another and that macromolecules and antigens from the brain parenchyma can access the CSF and then drain into the cervical lymph nodes via the meningeal lymphatic vasculatures (and possibly additional routes).

\section{Generation of immune responses and immune privilege}

As outlined above, the available evidence suggests that antigens from the CNS can access the cervical lymph nodes, and from there potentially induce an immune response. It was recently demonstrated that induction of massive death of oligodendrocytes leads to the generation of autoreactive $\mathrm{T}$ cells that can indeed induce multiple sclerosis-like symptoms in mice (83). Furthermore, expression of a foreign antigen on oligodendrocytes induces $\mathrm{T}$ cell response in the draining lymph nodes (97). These findings demonstrate that brain antigens can drain into cervical lymph nodes and generate an immune response there. Such a response might devel- 
op more slowly, require a larger amount of antigen release, or need a secondary signal (like danger-or pathogen-associated molecular patterns [DAMPs or PAMPs]) to be drained alongside the antigens to be generated. These limitations may explain why allografts in the brain parenchyma survive longer.

Another scenario envisages the possible existence of mechanisms within the CNS to ensure that immune responses to CNS-draining antigens are limited, or at least tightly regulated, via established peripheral tolerance. It could be argued that the unique nature of lymphatic drainage from the CNS is indeed what drives the phenomenon of immune privilege. Lymphatic ECs are reportedly able to shape the immune response (98-100). Although most evidence comes from lymph node-resident lymphatic ECs, meningeal lymphatic ECs might have unique properties that induce and control immune tolerance; if so, the specific mechanism through which tolerance to CNS antigens is established might implicate the meningeal lymphatic vessels. The existence of these vessels, therefore, does not question the immune privilege but rather mechanistically underlies it (refs. 101, 102, and Figure 3).

In an alternative scenario, it is possible that cervical lymph nodes possess unique properties that modulate the immune response to CNS-drained antigens. As recently demonstrated, strategic placement of macrophages and dendritic cells in lymph node capsules positions them as major players in orchestrating the proper activation of $\mathrm{T}$ and $\mathrm{B}$ cells in the lymph nodes (103). It might be hypothesized that CNS-draining lymph nodes are capable of tolerizing or tuning down an immune response.

Accumulating evidence over decades of research has shown that drainage from the brain parenchyma and its surroundings does indeed occur under both normal and pathological conditions, and therefore cannot account for the status of immune privilege as originally defined. It remains for in-depth studies to investigate and eventually understand how CNS antigens induce their unique immune responses. Among the first questions to be addressed are those concerning the contribution of the different drainage routes to CNS function under healthy and diseased conditions. We also suggest that the cervical lymph nodes should be examined and compared with those of peripheral systems as a critical step toward deciphering the specific mechanisms of CNS immune responses.

\section{Glymphatic-lymphatic connection: final remarks and take-home messages}

Finally, it is important to summarize several facts about the meningeal lymphatic and glymphatic systems, to emphasize their connection, and to highlight current gaps of knowledge and where the research in this field should be headed.

First, although a potential lymphatic connection between the CNS and periphery has been previously proposed, and although some works indicated a possible presence of lymphatic vessels in the meninges $(47,55-59,61)$ and even in the CNS parenchyma (104), recent publications using state-of-the-art microscopy have molecularly characterized and assessed the function of the meningeal lymphatic vessels in greater depth $(45,46)$. The presence of these vessels in different species, their precise location within the meningeal layers, and their accessibility by the CSF need further investigation.
Second, the discovery of meningeal lymphatic vessels does not question the mere presence of CNS immune privilege (or what would be more precisely termed "immune uniqueness"). This discovery certainly challenges the dogma that CNS immune privilege is in part due to the absence of CNS lymphatic drainage. Based on lessons mostly learned from tumor immunology $(99,100)$, meningeal lymphatic vessels may, in fact, provide a plausible mechanistic explanation for how CNS tolerance is established. Understanding how meningeal lymphatic vessels contribute to a unique immune status toward CNS-derived antigens demands intensive further investigation.

Third, multiple routes are proposed to allow the circulation of immune cells from the CNS to the periphery (meningeal lymphatics, refs. 45, 101; cribriform plate, refs. 87, 88; and rostral migratory stream, ref. 86). In-depth analysis of the contribution and functions of the different paths under both normal and pathological conditions is necessary to fully understand how the immune system senses the CNS and how each route might differentially contribute to generating and maintaining immune responses toward the CNS.

Fourth, the glymphatic system has been demonstrated to be responsible for movement of the CSF through the brain parenchyma (15). CSF moves into brain specifically within periarterial spaces and interchanges with ISF owing to the presence of AQP4 water channels in perivascular astrocyte end-foot processes. ISF then drains from brain within perivenous channels, again with AQP4 decreasing the resistance to fluid flow (15). Bulk flow of CSF and ISF within this pathway, driven by cerebral arterial pulsatility and sleep $(20,27)$, has been shown to be critical for the elimination of interstitial waste products such as $\mathrm{A} \beta$ (15). This is the only plausible system that would explain the abundant presence of CNSderived molecules within the CSF. Previous works arguing that ISF and interstitial solutes are drained via a periarterial intramural pathway $(30,32)$ are misleading because they are performed ex vivo. Therefore, they provide little dynamic information regarding ISF flows, making it difficult to conclude that periarterial spaces are the most proximal site of ISF drainage. It is likely that periarterial solute accumulation is the result of solute recirculation into brain from the CSF compartment. Additionally, as these studies often lack quantitation, it is unknown whether periarterial clearance is a representative or sporadic phenomenon.

It should be noted, however, that other clearance systems have been implicated in the removal of protein, notably $A \beta$, from the brain parenchyma. Indeed, studies report transvascular clearance of $A \beta$ primarily via LDL receptor-related protein 1 but also its removal by pericytes and myeloid cells (105-111). All these systems, including glymphatic and lymphatic drainage, must work in symbiosis to ensure the proper removal of protein. Thus, additional studies must address the physiological and pathophysiological interactions of the brain's clearance systems.

Fifth, the connection between the glymphatic system and meningeal lymphatic drainage has been demonstrated (46). This continuous flow of CSF that perfuses the brain parenchyma and is drained to cervical lymph nodes provides a plausible route to study CNS-immune connection $(101,102)$. Future studies should strengthen the functional connection between the glymphatic flow and the meningeal lymphatic drainage. Moreover, hypo- or 
hyperfunction of this connection may underlie neuroinflammatory and neurodegenerative disorders (especially those associated with protein accumulation). Function and dysfunction of meningeal lymphatic vessels and their connection to the glymphatic system should be addressed by future works.

Identification of meningeal lymphatic vessels and of the glymphatic system has reinvigorated the study of CNS immune privilege, its drainage, and fluid flow/perfusion through the CNS parenchyma. Further works should aim at better understanding of the connection between the two systems and their malfunction in neurological conditions. This complex yet mechanistically solid system could provide new therapeutic targets for neurological disorders associated with immune malfunction and/or protein aggregation, potentially leading to new therapies.

\section{Acknowledgments}

We thank A. Impagliazzo for the contributions to figure development and S. Smith for editing sections of the manuscript. This work was supported by grants from the NIH (AG034113 and NS096967) and the National Multiple Sclerosis Society to JK; from Jane and Aatos Erkko Foundation, the European Research Coun- cil (ERC) under the European Union's Horizon 2020 research and innovation program under grant agreement 743155, the Academy of Finland Centre of Excellence Program 2014-2019 (271845), and the Sigrid Juselius Foundation (all to KA). SA has been supported by Biomedicum Helsinki Foundation, The Finnish Medical Foundation, Orion Research Foundation, and Finnish Cultural Foundation. This work was also supported by the NIH (R01NS100366 and R01AG048769) and the Novo Nordisk Research Foundation to MN, and by the LE\&RN Postdoctoral Fellowship Award (to AL).

Address correspondence to Kari Alitalo, Wihuri Research Institute and Molecular Cancer Biology Program, Biomedicum Helsinki, 00014 University of Helsink, Haartmaninkatu 8, Helsinki, Finland. Phone: 358.9.191.25511; Email: kari.alitalo@helsinki.fi. Or to: Maiken Nedergaard, Center of Translational Neuromedicine, University of Rochester Medical School, 609 Elmwood Avenue, Rochester, New York 14642, USA. Phone: 585.478.0610; Email: Maiken_Nedergaard@URMC.Rochester.edu. Or to: Jonathan Kipnis, 409 Lane Rd, MR4 Building, Department of Neuroscience, University of Virginia, Charlottesville, Virginia 22908, USA. Phone: 434.983.3858; Email: kipnis@virginia.edu.
1. Oliver G. Lymphatic vasculature development. Nature Reviews Immunology. 2004;4(1):35-45.

2. Aspelund A, Robciuc MR, Karaman S, Makinen $\mathrm{T}$, Alitalo K. Lymphatic system in cardiovascular medicine. Circ Res. 2016;118(3):515-530.

3. Zhao Z, Nelson AR, Betsholtz C, Zlokovic BV. Establishment and dysfunction of the bloodbrain barrier. Cell. 2015;163(5):1064-1078.

4. Hladky SB, Barrand MA. Fluid and ion transfer across the blood-brain and blood-cerebrospinal fluid barriers; a comparative account of mechanisms and roles. Fluids Barriers CNS. 2016;13(1):19

5. Trevaskis NL, Kaminskas LM, Porter CJ. From sewer to saviour - targeting the lymphatic system to promote drug exposure and activity. Nat Rev Drug Discov. 2015;14(11):781-803.

6. Damkier HH, Brown PD, Praetorius J. Cerebrospinal fluid secretion by the choroid plexus. Physiol Rev. 2013;93(4):1847-1892.

7. Hladky SB, Barrand MA. Mechanisms of fluid movement into, through and out of the brain: evaluation of the evidence. Fluids Barriers CNS. 2014;11(1):26.

8. Jessen NA, Munk AS, Lundgaard I, Nedergaard M. The glymphatic system: a beginner's guide. Neurochem Res. 2015;40(12):2583-2599.

9. Nedergaard M. Neuroscience. Garbage truck of the brain. Science. 2013;340(6140):1529-1530.

10. Cserr HF, Ostrach LH. Bulk flow of interstitial fluid after intracranial injection of blue dextran 2000. Exp Neurol. 1974;45(1):50-60.

11. Cserr HF, Cooper DN, Milhorat TH. Flow of cerebral interstitial fluid as indicated by the removal of extracellular markers from rat caudate nucleus. Exp Eye Res. 1977;25(suppl):461-473.

12. Cserr HF, Cooper DN, Suri PK, Patlak CS. Efflux of radiolabeled polyethylene glycols and albumin from rat brain. Am J Physiol. 1981;240(4):F319-F328.

13. Rennels ML, Gregory TF, Blaumanis OR, Fujimoto K, Grady PA. Evidence for a 'paravascular' fluid circulation in the mammalian central nervous system, provided by the rapid distribution of tracer protein thoughout the brain from the subarachnoid space. Brain Res. 1985;326(1):47-63.

14. Rennels ML, Blaumanis OR, Grady PA. Rapid solute transport throughout the brain via paravascular fluid pathways. Adv Neurol. 1990;52:431-439.

15. Iliff JJ, et al. A paravascular pathway facilitates CSF flow through the brain parenchyma and the clearance of interstitial solutes, including amyloid B. Sci Transl Med. 2012;4(147):147ra111.

16. Mathiisen TM, Lehre KP, Danbolt NC, Ottersen OP. The perivascular astroglial sheath provides a complete covering of the brain microvessels: an electron microscopic 3D reconstruction. Glia. 2010;58(9):1094-1103.

17. Lundgaard I, et al. Direct neuronal glucose uptake heralds activity-dependent increases in cerebral metabolism. Nat Commun. 2015;6:6807.

18. Achariyar TM, et al. Glymphatic distribution of CSF-derived apoE into brain is isoform specific and suppressed during sleep deprivation. $\mathrm{Mol}$ Neurodegener. 2016;11(1):74.

19. Rangroo Thrane V, et al. Paravascular microcirculation facilitates rapid lipid transport and astrocyte signaling in the brain. Sci Rep. 2013;3:2582.

20. Xie L, et al. Sleep drives metabolite clearance from the adult brain. Science. 2013;342(6156):373-377.

21. Lundgaard I, et al. Glymphatic clearance controls state-dependent changes in brain lactate concentration. JCereb Blood Flow Metab. 2017;37(6):2112-2124.

22. Kress BT, et al. Impairment of paravascular clearance pathways in the aging brain. Ann Neurol. 2014;76(6):845-861.

23. Peng W, et al. Suppression of glymphatic fluid transport in a mouse model of Alzheimer's disease. Neurobiol Dis. 2016;93:215-225.

24. Gaberel T, et al. Impaired glymphatic perfusion after strokes revealed by contrast-enhanced MRI: a new target for fibrinolysis? Stroke. 2014;45(10):3092-3096.

25. Wang M, et al. Focal solute trapping and global glymphatic pathway impairment in a murine model of multiple microinfarcts. J Neurosci. 2017;37(11):2870-2877.

26. Iliff JJ, et al. Impairment of glymphatic pathway function promotes tau pathology after traumatic brain injury. J Neurosci. 2014;34(49):16180-16193.

27. Iliff JJ, et al. Cerebral arterial pulsation drives paravascular CSF-interstitial fluid exchange in the murine brain. J Neurosci. 2013;33(46):18190-18199.

28. Lee $\mathrm{H}$, et al. The effect of body posture on brain glymphatic transport. J Neurosci. 2015;35(31):11034-11044.

29. Auriel E, Greenberg SM. The pathophysiology and clinical presentation of cerebral amyloid angiopathy. Curr Atheroscler Rep. 2012;14(4):343-350.

30. Carare RO, et al. Solutes, but not cells, drain from the brain parenchyma along basement membranes of capillaries and arteries: significance for cerebral amyloid angiopathy and neuroimmunology. Neuropathol Appl Neurobiol.2008;34(2):131-144.

31. Bedussi B, et al. Enhanced interstitial fluid drainage in the hippocampus of spontaneously hypertensive rats. Sci Rep. 2017;7(1):744.

32. Bakker EN, et al. Lymphatic clearance of the brain: perivascular, paravascular and significance for neurodegenerative diseases. Cell Mol Neurobiol. 2016;36(2):181-194.

33. Engelhardt B, Vajkoczy P, Weller RO. The movers and shapers in immune privilege of the CNS. Nat Immunol. 2017;18(2):123-131.

34. Shinkai Y, et al. Amyloid $\beta$-proteins 1-40 and 1-42(43) in the soluble fraction of extraand intracranial blood vessels. Ann Neurol. 1995;38(3):421-428.

35. Carare RO, Hawkes CA, Jeffrey M, Kalaria RN, Weller RO. Review: cerebral amyloid angiopathy, 
prion angiopathy, CADASIL and the spectrum of protein elimination failure angiopathies (PEFA) in neurodegenerative disease with a focus on therapy. Neuropathol Appl Neurobiol. 2013;39(6):593-611.

36. Sharp MK, Diem AK, Weller RO, Carare RO. Peristalsis with oscillating flow resistance: a mechanism for periarterial clearance of amyloid $\beta$ from the brain. Ann Biomed Eng. 2016;44(5):1553-1565.

37. Coloma M, Schaffer JD, Carare RO, Chiarot PR, Huang P. Pulsations with reflected boundary waves: a hydrodynamic reverse transport mechanism for perivascular drainage in the brain. JMath Biol. 2016;73(2):469-490.

38. Asgari M, de Zélicourt D, Kurtcuoglu V. Glymphatic solute transport does not require bulk flow. Sci Rep. 2016;6:38635.

39. Alitalo K. The lymphatic vasculature in disease. Nat Med. 2011;17(11):1371-1380.

40. Secker GA, Harvey NL. VEGFR signaling during lymphatic vascular development: From progenitor cells to functional vessels. Dev Dyn. 2015;244(3):323-331.

41. Andres KH, von Düring M, Muszynski K, Schmidt RF. Nerve fibres and their terminals of the dura mater encephali of the rat. Anat Embryol. 1987;175(3):289-301.

42. Gausas RE, Daly T, Fogt F. D2-40 expression demonstrates lymphatic vessel characteristics in the dural portion of the optic nerve sheath. Ophthal Plast Reconstr Surg. 2007;23(1):32-36.

43. Furukawa M, Shimoda H, Kajiwara T, Kato S, Yanagisawa S. Topographic study on nerveassociated lymphatic vessels in the murine craniofacial region by immunohistochemistry and electron microscopy. Biomed Res. 2008;29(6):289-296.

44. Tarasoff-Conway JM, et al. Clearance systems in the brain-implications for Alzheimer disease. Nat Rev Neurol. 2015;11(8):457-470.

45. Louveau A, et al. Structural and functional features of central nervous system lymphatic vessels. Nature. 2015;523(7560):337-341.

46. Aspelund A, et al. A dural lymphatic vascular system that drains brain interstitial fluid and macromolecules. JExp Med. 2015;212(7):991-999.

47. Koh L, Zakharov A, Johnston M. Integration of the subarachnoid space and lymphatics: is it time to embrace a new concept of cerebrospinal fluid absorption? Cerebrospinal Fluid Res. 2005;2:6.

48. Carare RO, et al. Solutes, but not cells, drain from the brain parenchyma along basement membranes of capillaries and arteries: significance for cerebral amyloid angiopathy and neuroimmunology. Neuropathol Appl Neurobiol. 2008;34(2):131-144.

49. Szentistványi I, Patlak CS, Ellis RA, Cserr HF. Drainage of interstitial fluid from different regions of rat brain. Am J Physiol. 1984; 246(6 pt 2):F835-F844.

50. Pollay M. The function and structure of the cerebrospinal fluid outflow system. Cerebrospinal Fluid Res. 2010;7:9.

51. Kido DK, Gomez DG, Pavese AM Jr, Potts DG. Human spinal arachnoid villi and granulations. Neuroradiology. 1976;11(5):221-228.

52. Go KG, Houthoff HJ, Hartsuiker J, Blaauw EH, Havinga P. Fluid secretion in arachnoid cysts as a clue to cerebrospinal fluid absorption at the arachnoid granulation. JNeurosurg. 1986;65(5):642-648.

53. Upton ML, Weller RO. The morphology of cerebrospinal fluid drainage pathways in human arachnoid granulations. J Neurosurg. 1985;63(6):867-875.

54. Tripathi R. Tracing the bulk outflow route of cerebrospinal fluid by transmission and scanning electron microscopy. Brain Res. 1974;80(3):503-506.

55 . His W. Ueber ein perivaskulaeres Kanalsystem in den nervoesen Central-Organen und ueber dessen Beziehungen zum. Lymphsystem Z Wiss Zool. 1865;15:127-141.

56. Schwalbe G. Die Arachnoidalraum, ein Lymphraum und sein Zusammenhang mit den Perichorioidalraum. Zentralbl Med Wiss. 1869;7:465-467.

57. Cserr HF, Knopf PM. Cervical lymphatics, the blood-brain barrier and the immunoreactivity of the brain: a new view. Immunol Today. 1992;13(12):507-512.

58. Weller RO, Djuanda E, Yow HY, Carare RO. Lymphatic drainage of the brain and the pathophysiology of neurological disease. Acta Neuropathol. 2009;117(1):1-14.

59. Cserr HF, Harling-Berg CJ, Knopf PM. Drainage of brain extracellular fluid into blood and deep cervical lymph and its immunological significance. Brain Pathol. 1992;2(4):269-276.

60. Miura M, Kato S, von Lüdinghausen M. Lymphatic drainage of the cerebrospinal fluid from monkey spinal meninges with special reference to the distribution of the epidural lymphatics. Arch Histol Cytol. 1998;61(3):277-286.

61. Kida S, Pantazis A, Weller RO. CSF drains directly from the subarachnoid space into nasal lymphatics in the rat. Anatomy, histology and immunological significance. Neuropathol Appl Neurobiol. 1993;19(6):480-488.

62. Kaminski M, Bechmann I, Pohland M, Kiwit J, Nitsch R, Glumm J. Migration of monocytes after intracerebral injection at entorhinal cortex lesion site. J Leukoc Biol. 2012;92(1):31-39.

63. Johnston M, Zakharov A, Papaiconomou C, Salmasi G, Armstrong D. Evidence of connections between cerebrospinal fluid and nasal lymphatic vessels in humans, non-human primates and other mammalian species. Cerebrospinal Fluid Res. 2004;1(1):2.

64. Brierley JB, Field EJ. The connexions of the spinal sub-arachnoid space with the lymphatic system. J Anat. 1948;82(3):153-166.

65. Kwon S, Janssen CF, Velasquez FC, Sevick-Muraca EM. Fluorescence imaging of lymphatic outflow of cerebrospinal fluid in mice [published online ahead of print June 22, 2017]. JImmunol Methods. https://doi.org/10.1016/j.jim.2017.06.010.

66. Mollanji R, Bozanovic-Sosic R, Zakharov A, Makarian L, Johnston MG. Blocking cerebrospinal fluid absorption through the cribriform plate increases resting intracranial pressure. Am J Physiol Regul Integr Comp Physiol. 2002;282(6):R1593-R1599.

67. Gomez DG, Ehrmann JE, Gordon Potts D, Pavese $\mathrm{AM}$, Gilanian A. The arachnoid granulations of the newborn human: an ultrastructural study. Int J Dev Neurosci. 1983;1(2):139-147.

68. Papaiconomou C, Bozanovic-Sosic R, Zakharov
A, Johnston M. Does neonatal cerebrospinal fluid absorption occur via arachnoid projections or extracranial lymphatics? Am J Physiol Regul Integr Comp Physiol. 2002;283(4):R869-R876.

69. Johanson CE, Duncan JA, Klinge PM, Brinker T, Stopa EG, Silverberg GD. Multiplicity of cerebrospinal fluid functions: New challenges in health and disease. Cerebrospinal Fluid Res. 2008;5:10.

70. Rubenstein E. Relationship of senescence of cerebrospinal fluid circulatory system to dementias of the aged. Lancet. 1998;351(9098):283-285.

71. Medawar PB. Immunity to homologous grafted skin; the relationship between the antigens of blood and skin. Br JExp Pathol. 1946;27:15-24.

72. Mason DW, Charlton HM, Jones AJ, Lavy CB, Puklavec M, Simmonds SJ. The fate of allogeneic and xenogeneic neuronal tissue transplanted into the third ventricle of rodents. Neuroscience. 1986;19(3):685-694.

73. Nicholas MK, Antel JP, Stefansson K, Arnason BG. Rejection of fetal neocortical neural transplants by H-2 incompatible mice. J Immunol. 1987;139(7):2275-2283.

74. Derecki NC, et al. Regulation of learning and memory by meningeal immunity: a key role for IL-4. J Exp Med. 2010;207(5):1067-1080.

75. Kipnis J. Multifaceted interactions between adaptive immunity and the central nervous system. Science. 2016;353(6301):766-771.

76. Ransohoff RM, Engelhardt B. The anatomical and cellular basis of immune surveillance in the central nervous system. Nat Rev Immunol. 2012;12(9):623-635.

77. Shechter R, London A, Schwartz M. Orchestrated leukocyte recruitment to immune-privileged sites: absolute barriers versus educational gates. Nat Rev Immunol. 2013;13(3):206-218.

78. Schläger C, et al. Effector T-cell trafficking between the leptomeninges and the cerebrospinal fluid. Nature. 2016;530(7590):349-353.

79. Hatterer E, et al. How to drain without lymphatics? Dendritic cells migrate from the cerebrospinal fluid to the B-cell follicles of cervical lymph nodes. Blood. 2006;107(2):806-812.

80. van Zwam M, et al. Surgical excision of CNS-draining lymph nodes reduces relapse severity in chronic-relapsing experimental autoimmune encephalomyelitis. JPathol. 2009;217(4):543-551.

81. de Vos AF, et al. Transfer of central nervous system autoantigens and presentation in secondary lymphoid organs. JImmunol. 2002;169(10):5415-5423.

82. Locatelli G, et al. Primary oligodendrocyte death does not elicit anti-CNS immunity. Nat Neurosci. 2012;15(4):543-550.

83. Traka M, Podojil JR, McCarthy DP, Miller SD, Popko B. Oligodendrocyte death results in immune-mediated CNS demyelination. Nat Neurosci. 2016;19(1):65-74.

84. Urra X, Miró F, Chamorro A, Planas AM. Antigen-specific immune reactions to ischemic stroke. Front Cell Neurosci. 2014;8:278.

85. Pappolla M, Sambamurti K, Vidal R, PachecoQuinto J, Poeggeler B, Matsubara E. Evidence for lymphatic $A \beta$ clearance in Alzheimer's transgenic mice. Neurobiol Dis. 2014;71:215-219.

86. Mohammad MG, et al. Immune cell trafficking 
from the brain maintains CNS immune tolerance. JClin Invest. 2014;124(3):1228-1241.

87. Goldmann J, Kwidzinski E, Brandt C, Mahlo J, Richter D, Bechmann I. T cells traffic from brain to cervical lymph nodes via the cribroid plate and the nasal mucosa. JLeukoc Biol. 2006;80(4):797-801.

88. Kaminski M, Bechmann I, Pohland M, Kiwit J, Nitsch R, Glumm J. Migration of monocytes after intracerebral injection at entorhinal cortex lesion site. J Leukoc Biol. 2012;92(1):31-39.

89. Clarkson BD, et al. CCR7 deficient inflammatory dendritic cells are retained in the central nervous system. Sci Rep. 2017;7:42856.

90. Bauer J, et al. T-cell apoptosis in inflammatory brain lesions: destruction of $\mathrm{T}$ cells does not depend on antigen recognition. Am J Pathol. 1998;153(3):715-724.

91. Choi C, Benveniste EN. Fas ligand/Fas system in the brain: regulator of immune and apoptotic responses. Brain Res Brain Res Rev. 2004;44(1):65-81.

92. Schiefenhövel F, Immig K, Prodinger C, Bechmann I. Indications for cellular migration from the central nervous system to its draining lymph nodes in CD11c-GFP(+) bone-marrow chimeras following EAE. Exp Brain Res. 2017;235(7):2151-2166.

93. Spector R, Johanson CE. Vitamin transport and homeostasis in mammalian brain: focus on Vitamins B and E. J Neurochem. 2007;103(2):425-438.

94. Spector R, Robert Snodgrass S, Johanson CE. A balanced view of the cerebrospinal fluid compo- sition and functions: Focus on adult humans. Exp Neurol. 2015;273:57-68.

95. Brombacher TM, et al. IL-13-mediated regulation of learning and memory. J Immunol. 2017;198(7):2681-2688.

96. Filiano AJ, et al. Unexpected role of interferon- $\gamma$ in regulating neuronal connectivity and social behaviour. Nature. 2016;535(7612):425-429.

97. Harris MG, et al. Immune privilege of the CNS is not the consequence of limited antigen sampling. Sci Rep. 2014;4:4422.

98. Betterman KL, Harvey NL. The lymphatic vasculature: development and role in shaping immunity. Immunol Rev. 2016;271(1):276-292.

99. Card CM, Yu SS, Swartz MA. Emerging roles of lymphatic endothelium in regulating adaptive immunity. J Clin Invest. 2014;124(3):943-952.

100.Randolph GJ, Ivanov S, Zinselmeyer BH, Scallan JP. The lymphatic system: integral roles in immunity. Annu Rev Immunol. 2017;35:31-52.

101. Louveau A, Da Mesquita S, Kipnis J. Lymphatics in neurological disorders: a neuro-lympho-vascular component of Multiple Sclerosis and Alzheimer's disease? Neuron. 2016;91(5):957-973.

102. Louveau A, Harris TH, Kipnis J. Revisiting the mechanisms of CNS immune privilege. Trends Immunol. 2015;36(10):569-577.

103. Gerner MY, Torabi-Parizi P, Germain RN. Strategically localized dendritic cells promote rapid $\mathrm{T}$ cell responses to lymph-borne particulate antigens. Immunity. 2015;42(1):172-185.
104.Prineas JW. Multiple sclerosis: presence of lymphatic capillaries and lymphoid tissue in the brain and spinal cord. Science. 1979;203(4385):1123-1125.

105. Deane R, Sagare A, Zlokovic BV. The role of the cell surface LRP and soluble LRP in blood-brain barrier A $\beta$ clearance in Alzheimer's disease. Curr Pharm Des. 2008;14(16):1601-1605.

106.Zlokovic BV, Deane R, Sagare AP, Bell RD, Winkler EA. Low-density lipoprotein receptorrelated protein-1: a serial clearance homeostatic mechanism controlling Alzheimer's amyloid $\beta$-peptide elimination from the brain. J Neurochem. 2010;115(5):1077-1089.

107. Deane R, et al. LRP/amyloid $\beta$-peptide interaction mediates differential brain efflux of $A \beta$ isoforms. Neuron. 2004;43(3):333-344.

108. Bell RD, et al. Transport pathways for clearance of human Alzheimer's amyloid $\beta$-peptide and apolipoproteins $\mathrm{E}$ and $\mathrm{J}$ in the mouse central nervous system. JCereb Blood Flow Metab. 2007;27(5):909-918.

109. Deane R, et al. apoE Isoform-specific disruption of amyloid beta peptide clearance from mouse brain. J Clin Invest. 2008;118(12):4002-4013.

110.Storck SE, et al. Endothelial LRP1 transports amyloid- $\beta(1-42)$ across the blood-brain barrier. JClin Invest. 2016;126(1):123-136.

111. Sagare AP, et al. Pericyte loss influences Alzheimer-like neurodegeneration in mice. Nat Commun. 2013;4:2932. 